\title{
Coexistence between IEEE802.15.4 and IEEE802.11 through cross-technology signaling
}

\author{
Jan Bauwens, Bart Jooris, Peter Ruckebusch, Domenico Garlisi, Josesph Szurley, Marc Moonen, \\ Spilios Giannoulis, Ingrid Moerman, Eli De Poorter \\ Email: jan.bauwens@intec.ugent.be \\ Ghent University - imec \\ IDLab \\ iGent Tower - Department of Information Technology \\ Technologiepark-Zwijnaarde 15, B-9052 Ghent, Belgium
}

\begin{abstract}
When different technologies use the same frequency bands in close proximity, the resulting interference typically results in performance degradation. Coexistence methods exist, but these are often technology specific and requiring technology specific interference detection methods. To remove the root cause of the performance degradation, devices should be able to negotiate medium access even when using different technologies. To this end, this paper proposes an architecture that allows crosstechnology medium access by means of a Time Division Multiple Access (TDMA) scheme. In order to achieve cross-technology synchronization, which is required for the TDMA solution, an energy pattern beacon is transmitted. The use of energy patterns is sufficiently technology agnostic to allow multiple technologies to negotiate between each other. The feasibility of the solution is experimentally demonstrated in a large scale testbed using 50 IEEE802.15.4 and IEEE802.11 devices, demonstrating a successful cross-technology TDMA synchronization rate of over $90 \%$.

Index Terms-MAC protocols, interference mitigation, crosstechnology TDMA scheme, energy pattern beacon, parameter negotiation
\end{abstract}

\section{INTRODUCTION}

Currently a significant number of wireless technologies use the unlicensed $2.4 \mathrm{GHz}$ Industrial, Scientific and Medical (ISM) frequency bands for wireless communication. The increasing number of devices including laptops, smartphones, IEEE802.15.4 nodes, bluetooth devices, etc. using the same limited medium has caused a large number of interference issues. Without medium access negotiation, a throughput loss of up to $30 \%$ for IEEE802.11 and $60 \%$ for IEEE802.15.4 is possible when both technologies coexist in the same environment [3]. Within the same technology interference can easily be minimized by designing medium access control protocols (MAC's) that intelligently allocate the medium to individual devices. For cross-technology MAC protocols the choices are limited: agreements on medium usage between different technologies is difficult due to a lack of direct communication possibilities between devices. Several attempts were made at creating a more advanced architecture that allows cross-technology communication. These architectures were not scalable due to overhead, or were limited to only a couple of technologies [1], [2].
In this paper an architecture is proposed that combines the use of energy detection and a backbone for cross-technology communication between IEEE802.11 and IEEE802.15.4 devices which results in fair use of the limited wireless medium. The focus lies on IEEE802.11 and IEEE802.15.4, but the concepts and architecture described are also applicable for other technologies.

The contributions of this paper are as follows:

1) cross-technology synchronization phase based on energy detection;

2) cross-technology communication which enables channel access control in different technologies;

3) multi-platform set-up and evaluation of the solution in a large scale testbed.

The paper is organized as follows. First section 2 overviews the current state of the art in terms of minimizing crosstechnology interference. Afterwards, section 3 gives an overview of how the cross-technology TDMA scheme proposed in this paper can be used for minimizing crosstechnology interference. Next, section 4 explains the crosstechnology controller architecture and how it is vital for the usage in the TDMA protocol. This is followed by section 5, which is a general evaluation of the solution. Finally, section 6 concludes the paper.

\section{STATE OF THE ART}

This section gives a short overview of related work that aims to improve cross-technology coexistence. The technique that is easiest to implement, and thus most commonly used, is selecting channels to achieve minimal frequency overlap by different technologies. This solution consists of detecting channel loads and choosing the 'best channel' accordingly [4]. Due to the increasing number of devices using the same wireless medium it becomes less feasible to select a channel that has a lower degree of occupancy. Choosing a better channel is also not an obvious choice due to the unpredictable nature of the wireless medium and its channel usage.

Attempts at creating a model of white space in a wireless medium have been made. A node use of this model to access the medium at the most optimal moments [5]. Unfortunately 
the same problem arises as when changing channel by detecting channel load: medium access is highly unpredictable and can change from one moment to another making the use of a model, in most cases, too unreliable.

Several research groups make use of energy detection mechanisms in their architectures. ESense [6] proposes an alphabet system based on packet length for unidirectional communication between different wireless technologies. Each character in the alphabet is mapped on a specific packet length. Detecting the packet length is done by measuring the time difference between the rising and downward flank of the energy pattern. Subsequently, based on the measured packet duration, the corresponding character is determined. Having a large alphabet raises problems with uniqueness: due to the usage of packet length and not a real 'pattern', a regular data packet might be mistaken for a cross-technology packet. To counter this the cross-technology packet is repeated several times, which results in a wastage of channel time in a medium that already might be saturated. WizSync [7] is a second system that makes use of energy detection but contrary to the concepts described in this paper, existing IEEE802.11 beacons are used which are created by default access points. The major advantage of this technology is that it makes use of existing hardware drivers, the drawback that it results in a fixed schedule due to the lack of control over the beacon interval. In a third solution [8] the medium is statically divided between IEEE802.11 and IEEE802.15.4 nodes. Within their own time frame the nodes can use a technology specific MAC protocol (e.g. CSMA in IEEE802.11 and TSCH in IEEE802.15.4). The IEEE802.11 nodes start a timer when a IEEE802.15.4 packet is detected, the nodes will reactivate after this timer has stopped. This solution lacks scalability options as the configuration is static during the whole lifetime of the devices. This paper improves upon the above concepts by using unique cross-technology energy beacons that allow to reconfigure the devices after deployment.

\section{CROSS-TECHNOLOGY INTERFERENCE MITIGATION}

In order to achieve a fair distribution of the medium, a crosstechnology MAC protocol is necessary. Two kinds of MAC protocols can be distinguished: contention based and noncontention based. In a contention based approach, devices will sense the medium and if found idle will try and send a packet. If the medium is found occupied a back off will be performed before a new attempt can be made to transmit the packet. The problem with such a mechanism is twofold: the first issue arises from a differing transmission power (typically $0 \mathrm{dBm}$ for IEEE802.15.4, 20dBm for IEEE802.11). A IEEE802.11 node that is far enough to not sense the transmissions of a IEEE802.15.4 sensor node can start sending and blow away all the transmission attempts of the sensor node. A second issue results from the turnaround time of the radio $(128 \mu \mathrm{s}$ for IEEE802.15.4, 5 $\mu$ s for IEEE802.11), as the sensor node will always draw the shorter straw when trying to compete with a WiFi node [9]. As such contention based approaches mostly unfairly benefit IEEE802.11 devices.
A non-contention based approach will result in a fairer solution: each node gets it's own share of the medium during which only that node can access it. The most commonly used approach is Time Division Multiple Access (TDMA) where channel access is divided in time slots. To avoid transmission collisions, all nodes should be tightly synchronized to the same reference time which is typically kept by one or more central nodes. TDMA is a proven mechanism that can provide high throughput and is typically very robust. Despite the availability of a TDMA protocol in IEEE802.11 as well as in IEEE802.15.4, a cross-technology TDMA is not easy to implement due to a strong dependence on the distribution of timing information. This timing information can only be transmitted to nodes of the same technology and thus a novel approach needs to be taken to achieve synchronization between wireless technologies.

A radio chip might not be able to interpret a beacon from a different technology, but it can detect when the medium is being accessed by means of energy detection. Even more importantly it can detect when a switch between idle and occupied has occurred on a channel. This mechanism in combination with timing information can be used for synchronization purposes. A central node sends beacons which are simple energy patterns containing a sequence of channel access and idle periods with very specific timings.

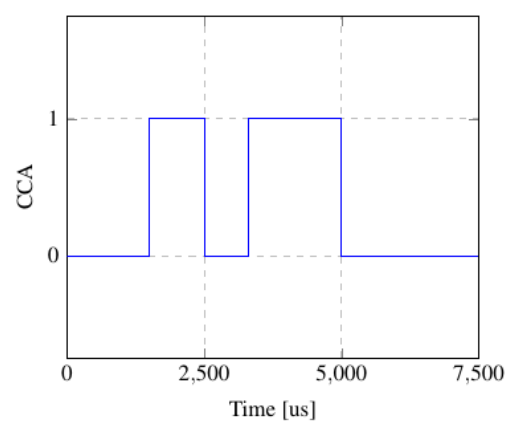

Fig. 1. Cross-technology beacon transmission

Different approaches from coding theory can be used to define custom beacons with different meanings allowing full cross-technology protocol implementations, e.g. to allow negotiation between different devices. Advanced implementations can even include error correction codes [10]. Figure 1 shows an example implementation of such a beacon slot used in the TDMA solution. It is of utmost importance that the pattern should be unique enough to not be labeled as interference, hence the up times shouldn't coincide with commonly used packet lengths [6]. For this implementation, following TDMA slot durations were used: a IEEE802.11 slot corresponds with a slot duration of $2500 \mu \mathrm{s}$, while the IEEE802.15.4 slot duration is $7500 \mu \mathrm{s}$. These durations were chosen by taking into account the maximum packet air time for each technology as well as the slot durations being a multiple of each other. The decision was made to use a IEEE802.11 device to generate the beacon for a number of reasons: (1) higher data rate which allows a 


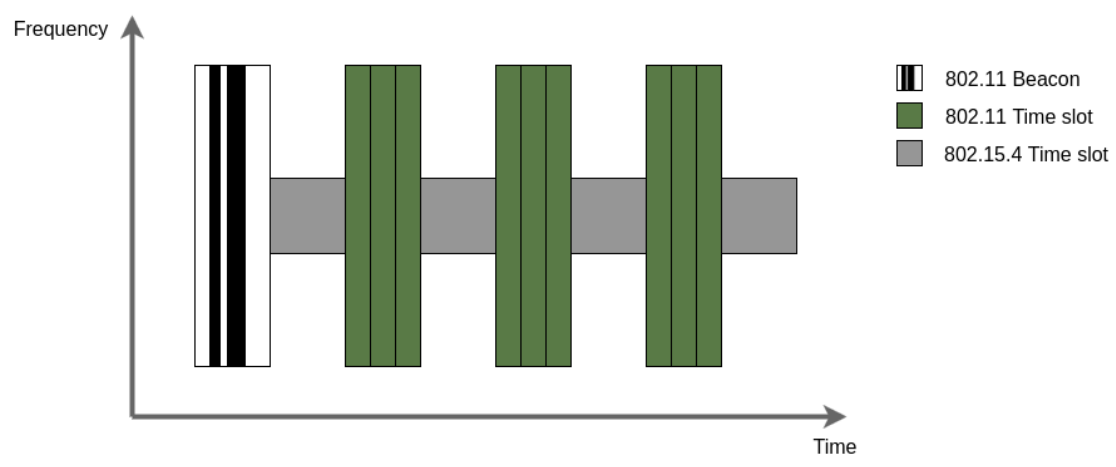

Fig. 2. Cross-technology synchronization and TMDA schedule. Each IEEE802.15.4 slot is followed by three IEEE802.11 slots

more fine-grained control of the beacon, (2) higher maximum transmission power $(100 \mathrm{~mW}$ or $20 \mathrm{dBm}$ to $1 \mathrm{~mW}$ or $0 \mathrm{dBm})$, (3) a single IEEE802.11 channel overlaps with nine bluetooth channels and four IEEE802.15.4 channels which allows to synchronize multiple channels with a single channel $\mathrm{WiFi}$ access point.

To allow other technologies synchronize on the IEEE802.11 beacon, clear channel assessment (CCA) is used. In general the CCA status is used as an indication if a particular channel is available for transmission or not. The CCA check is based on the received signal strength (RSSI) and a configurable threshold. An update is generated every 4 clock cycles (for IEEE802.15.4 nodes). The MAC protocol will be notified when there has been a change in status of the CCA accompanied by the time stamp on which this change has occurred. With some simple subtractions it can be calculated how long the CCA was high/low and if this corresponds with the beacon generated by the access point. Some margins have to be taken into account: a IEEE802.11 radio is more precise so there might be some variation on the measured beacon durations.

To minimize data loss a number of improvements can be used, for example it is not always necessary to synchronize on a beacon every superframe. Based on the amount of clock drift on a particular device in combination with the time since the last synchronization a deadline can be derived on which the node has to be synchronized again.

$$
\text { time_since_sync } * \text { clock_drift }=d r i f t<d r i f t \_m a x
$$

If it wasn't possible to distinguish a beacon within the synchronization slot of the superframe, but the deadline hasn't passed yet, execution will be continued as if the synchronization has succeeded. If the deadline has passed the node will inevitably have to wait until the beacon has been detected. With this easy technique it is possible to lower the CCA threshold and by consequence cover a larger area.

A second improvement are intra-technology synchronization nodes that are responsible to synchronize the nodes within their own network. These nodes, in their turn, synchronize on a central node in the same way that was described in previous sections. The synchronization nodes can be put in close proximity to the central node with a high CCA threshold thus minimizing the chance that interference will have an impact on synchronization. Nodes being synchronized within their own technology have the added advantage that they are less prone to interference.

\section{CROSS-TECHNOLOGY COMMUNICATION FOR MEDIUM ACCESS NEGOTIATION}

The previous section illustrated how a cross-technology TDMA solution can be used in order to achieve a fair distribution of the medium. In the example scheme (in figure 2) it was shown that the superframe contains a sequence of time slots where each of the IEEE802.11 slots was followed by a single IEEE802.15.4 slot. One could argue that the medium access is more or less fair by giving each technology half of the medium access time, but this static configuration might not always reflect the needs of the network. A dynamic slot structure will, in most cases, more optimally make use of the medium. For example a sensor device might not need a slot every superframe: the time allocated might be more suited to give to a node that, at that moment, needs more slots for being able to send all its data over the network. Such nodes need to request extra slots to send data in.

To allow for schedule negotiation the WiSHFUL architecture was used: this architecture supplies unified programming interfaces (UPI's) for local and remote protocol configuration and monitoring according to the WiSHFUL vision [11]. Using this architecture, nodes can be addressed independent of their originating technology using technology-independent, unified interfaces 1 .

In figure 3 a network of devices is displayed within the same collision domain. These devices are all connected via a WiSHFUL control network. At the top level a cross-technology controller can be found. It communicates with the different technology-specific controllers over a backbone network (to make network-wide changes spanning multiple technologies). In the context of this paper it is responsible for keeping track of time slot allocation for each technology without knowledge about specific node needs. It accepts requests from the technology specific network controllers for a change in

\footnotetext{
${ }^{1}$ Cross-technology negotiation could also be implemented using custom beacons with other energy patterns, at the cost of reduced available air-time.
} 


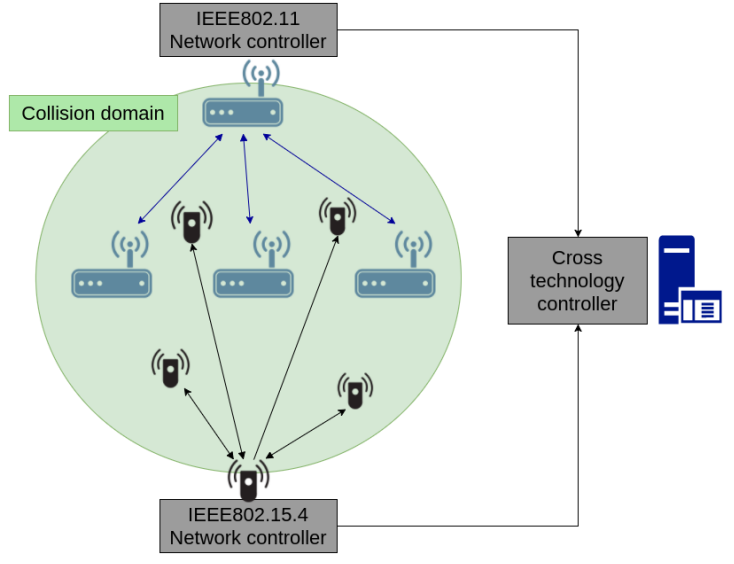

Fig. 3. Cross-technology controller framework

number of slots. A new slot layout is calculated which can subsequently be distributed to all technology specific network controllers.

A technology specific network controller acts as an intermediary between the cross-technology controller and the actual nodes. It keeps track of the nodes inside their network and their requirements and allocates available resources accordingly. These resources can be requested from the cross-technology controller. It should be noted that it is of utmost importance to not only request resources, but allocated resources should be released once they are not needed anymore. The last level of controller logic houses in the nodes itself. Depending on the application needs, the number of necessary resources varies. When allocated resources don't map correctly on the applications needs anymore, the node should ask the network controller to allocate resources differently.

\section{Evaluation}

The TDMA solution has been implemented and tested extensively. This section describes how these tests were conducted and which hardware was used.

\section{A. Used hardware and software}

The evaluation was performed in the imec w-iLab.t wireless testbed [14]. It allows for flexible testing of the functionality and performance of wireless networking protocols and systems in a time-effective way, by providing hardware and the means to install and configure firmware and software on (a selection of) nodes, schedule automated experiments, and collect, visualize and process results. This testbed is equipped with a large number of wireless nodes with IEEE $802.11 \mathrm{a} / \mathrm{b} / \mathrm{g} / \mathrm{n}$, IEEE802.15.4 and IEEE802.15.1 interfaces. The embedded PC's are connected by a wired interface for management purposes. A Linux distribution is available by default (although this can be changed). It is up to the user to define the behavior of the embedded PCs by installing software and/or scripts on the nodes. As such, the embedded PCs can be used for a very broad set of experiments. The testbed allows access to the embedded PCs individually via SSH (by a web-based testbed interface), so the binaries and scripts can be spread and installed on multiple nodes at once.

Following hardware was used to conduct the experiments:

- IEEE802.11: PC Engines ALIX;

- IEEE802.15.4: RM090 sensor node connected to a Zotac;

- Interference: Zotac with 802.11abgn Wifi card

The experiment orchestration was carried out with WiSHFUL local controllers running on the ALIX and Zotac devices, totalling 50 nodes spread throughout the testbed environment which covers $1200 \mathrm{~m}^{2}$. Each node was loaded with the TDMA solution. The global control program, which controls the different nodes and collects node statistics, can be run on the experimenters own device as well as one of the testbed nodes. For the experiments conducted in this paper, the global controller was run on a Zotac node inside the testbed. The results were logged from the global controller to a MySQL database. The control programs are publicly available ${ }^{2}$.

To evaluate the feasibility and performance of the solution described in this paper, two hardware platforms were modified. For the IEEE802.11 nodes the Wireless MAC Processor ${ }^{3}$ (WMP) was used [12]. The WMP implementation allows easy generation of the beacon pattern by generating packets of predefined length at specific moments. For the IEEE802.15.4 nodes, a TDMA has been implemented in TAISC ${ }^{4}$, which is an architecture for easy MAC protocol creation [13]. By modifying the existing TDMA protocols, it became possible to detect the energy beacon. It has to be noted that both the WMP, as well as TAISC, are open source. The final TDMA solution is given in figure 2 . A beacon slot is followed by the rest of the superframe in which a number of slots is divided among the different nodes in the network independent of which technology the node belongs to.

\section{B. Performance evaluation}

1) Analysis of the TDMA solution: Figure 4 shows the cross-technology TDMA protocol that results from the cross-technology negotiation between 50 IEEE802.15.4 and IEEE802.11 nodes. The figure is obtained by recording RSSI samples using a USRP node. The USRP was configured to listen on $2440 \mathrm{Mhz}$ which corresponds with channel 18 on IEEE802.15.4 and channel 6 on IEEE802.11. The beacon, which is being transmitted every $75 \mathrm{~ms}$, can clearly be distinguished by the two short consecutive peaks. Following the beacon is the rest of a superframe which consists of a number of IEEE802.11 and IEEE802.15.4 slots which are allocated to specific nodes. None of the nodes sends outside of its slot, by consequence the channel access is guaranteed. The resulting solution is a highly synchronized TDMA in which each node gets its own fair share of the medium.

2) Analysis of the synchronization: At first it was attempted to find the optimal CCA threshold to detect the energy beacon. For this purposes all nodes reported the number of synchronization beacons detected within a given time interval and with

\footnotetext{
${ }^{2} \mathrm{https} / / /$ github.com/wishful-project

${ }^{3}$ https://github.com/ict-flavia/Wireless-MAC-Processor

${ }^{4}$ https://github.com/WirelessTestbedsAcademy/wishful-contiki-tree
} 


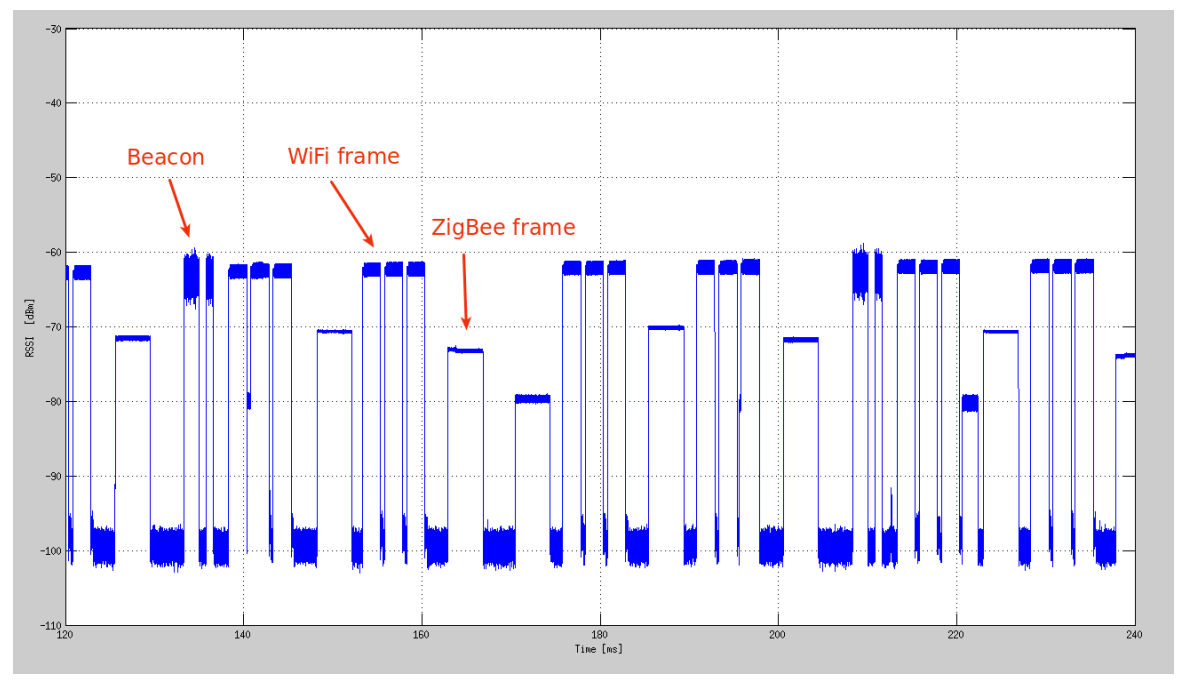

Fig. 4. Cross-technology synchronization and TMDA schedule

a given CCA threshold. Figure 5 shows the ratio of number of syncs detected relative to the number of transmitted beacons. If the CCA is chosen too low only nodes close to the beacon transmitter will be able to synchronize, if chosen too high the beacon might become indistinguishable from interference.

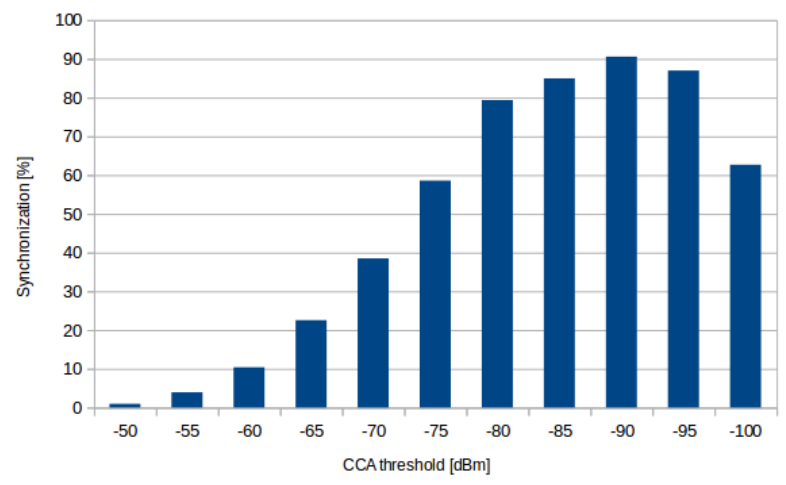

Fig. 5. Impact of CCA threshold on synchronization

For the two evaluated technologies the optimal CCA threshold is $-90 \mathrm{dBm}$ which allows for a synchronization success rate of $90.5 \%$. Since the clock drift is sufficiently low to ensure synchronized operation during multiple superframes, this synchronization rate is sufficient to allow robust long-term operation even when some cross-technology synchronization frames are not detected.

Another factor that impacts the quality of the TDMA solution is the distance to the beacon transmitter. Figure 6 shows that the synchronization decays linearly with distance to the transmitter. This is to be expected as the RSSI gets lower and thus the chances to get interfered get higher.

3) Analysis of the impact of external (non-controllable) interference: In an ideal world the solution proposed in this paper would be used on all possible devices and the possibility of external interference will be negated. Since not all devices

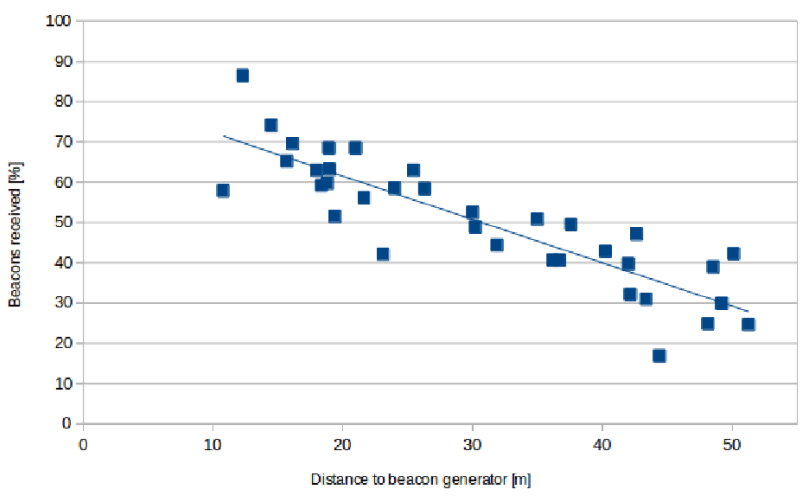

Fig. 6. Impact of distance on cross-technology synchronization success rate

will simultaneously make the switch to the proposed TDMA solution, external interference will likely still be present. The previous tests were conducted in a semi-shielded testbed facility, and as a result the influence of external interference was minimal. Interference/data originating from nodes outside the network has a big influence in the smooth functioning of the proposed solution. If such interference falls directly inside a beacon slot, nodes might not be able to distinguish the beacon. To successfully receive the energy pattern beacon, the CCA threshold should be set at an optimal value. If chosen too high the beacon will be labeled as noise on nodes that are further from the central node. Therefore, the CCA threshold has a direct impact on the size of the network that can be covered by a single beacon sender. If on the other hand the value is chosen too low, it might pick up energy not originating from nodes inside the network. The pattern can thus become indistinguishable from background noise. Consequently an ideal value does not exist and a trade-off has to be made between network size and the number of times the nodes can safely not receive the synchronization beacon.

To emulate the effects of interference a number of $\mathrm{WiFi}$ 

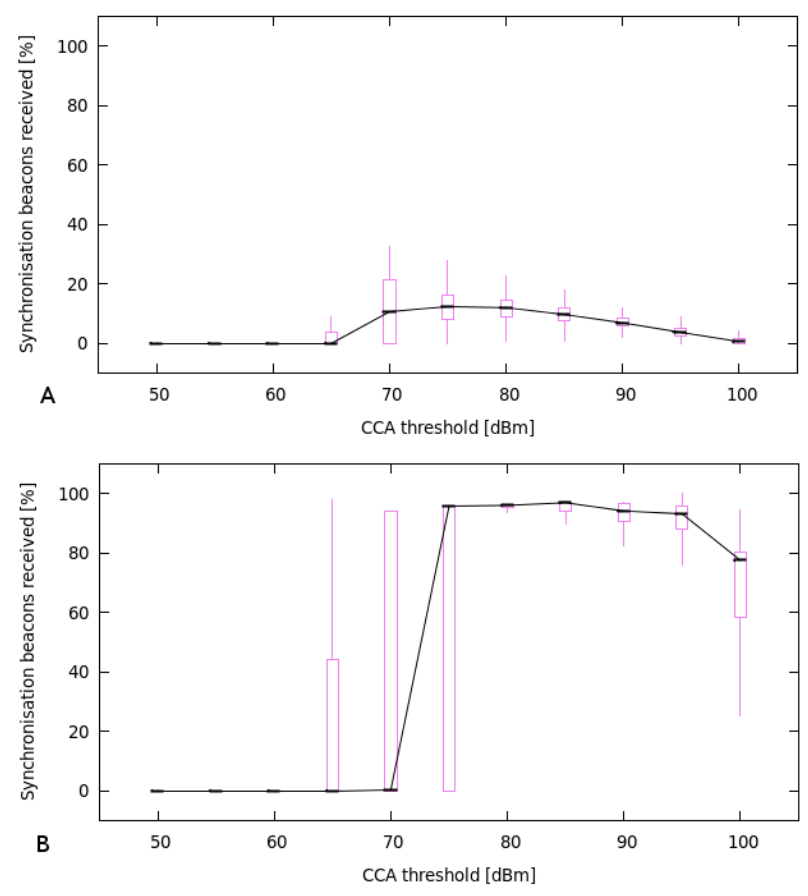

Fig. 7. CCA threshold impact on synchronization (a) without external interference and (b) with external interfence

nodes were selected throughout the environment which use the regular CSMA protocol as described in the IEEE802.11 standard and have no knowledge about the TDMA used in the other nodes. Figure 7 illustrates the effects of interference generated by the IEEE802.11 nodes. The optimal CCA threshold has shifted to $-75 \mathrm{dBm}$. The number of detected beacons has shrunk dramatically, so it can be concluded that non-controllable external interference is a big issue for the described solution.

The resulting work can be demonstrated in real-time, using the WiSHFUL Portable Testbed or the Wilab-t testbed facility. We hereby refer to the demo abstract for IEEE INFOCOM CENRT 2017 [15].

\section{CONCLUSION}

Cross-technology interference occurs when multiple devices use the same frequency bands without the possibility to successfully negotiate medium access. This paper focused around mitigating cross-technology interference by means of a simple TDMA mechanism based on an energy pattern beacon. It was shown to be possible to generate such a beacon in a way that other technologies were able to synchronize on it. A shared TDMA scheme was devised with a cross-technology synchronization phase. Initially the TDMA schedule was configured statically. To cope with dynamic application requirements, the solution was extended with a cross-technology management framework which enable channel access control in different technologies by dynamically allocating time slots to specific technologies/nodes. The cross-technology synchronization was experimentally tested on real hardware in the Wilab-t testbed facility. It was determined that the nodes were able to synchronize more than $90 \%$ of the time. In addition, optimal CCA thresshold values were determined both with and without external interference. The resulting solution allows for robust communication when the wireless medium is being shared by multiple technologies.

\section{ACKNOWLEDGMENT}

This work was partially supported by project SAMURAI: Software Architecture and Modules for Unified RAdIo control, and European Commission Horizon 2020 Programme under grant agreement no. 645274 (WiSHFUL).

\section{REFERENCES}

[1] R. Wang, R. Chang, and H. Chao. "Internetworking between ZigBee/802.15. 4 and IPv6/802.3 network." SIGCOMM Data Communication Festival (2007).

[2] P. Dutta, J. Hui, J. Jeong, S. Kim, C. Sharp, J. Taneja, G. Tolle, K. Whitehouse, and D. Culler. "Trio: enabling sustainable and scalable outdoor wireless sensor network deployments." Proceedings of the 5th international conference on Information processing in sensor networks. ACM, 2006.

[3] S. Pollin, M. Ergen, M. Timmers, A. Dejonghe, L. Van der Perre, F. Catthoor, I. Moerman, and A. Bahai. "Distributed cognitive coexistence of 802.15. 4 with IEEE802.11.”, 2006 1st International Conference on Cognitive Radio Oriented Wireless Networks and Communications. IEEE, 2006.

[4] M.S. Kang, J.W. Chong, H. Hyun, S.M. Kim, B.H. Jung, and D.K. Sung. "Adaptive interference-aware multi-channel clustering algorithm in a Zigbee network in the presence of WLAN interference." 2007 2nd International Symposium on Wireless Pervasive Computing. IEEE, 2007.

[5] J. Huang, G. Xing, G. Zhou, and R. Zhou. "'Beyond co-existence: Exploiting WiFi white space for Zigbee performance assurance." Network Protocols (ICNP), 2010 18th IEEE International Conference on. IEEE, 2010.

[6] K. Chebrolu, and A. Dhekne. "Esense: communication through energy sensing." Proceedings of the 15th annual international conference on Mobile computing and networking. ACM, 2009.

[7] T. Hao, R. Zhou, G. Xing, M.W. Mutka, and J. Chen. "Wizsync: Exploiting wi-fi infrastructure for clock synchronization in wireless sensor networks." IEEE Transactions on Mobile Computing 13.6 (2014): 13791392.

[8] P. De Valck, I. Moerman, D. Croce, F. Giuliano, I. Tinnirello, D. Garlisi, E. De Poorter, and B. Jooris. "Exploiting programmable architectures for WiFi/ZigBee inter-technology cooperation." EURASIP Journal on Wireless Communications and Networking 2014.1 (2014): 1-13.

[9] N.C. Tas, C. Sastry, and Z. Song. "IEEE 802.15. 4 throughput analysis under IEEE 802.11 interference." Proceedings of the International Symposium on Innovations and Real Time Applications of Distributed Sensor Networks. 2007.

[10] F.J. MacWilliams, and N.J.A. Sloane. The theory of error correcting codes. Elsevier, 1977

[11] C. Fortuna, P. Ruckebusch, C. Van Praet, I. Moerman, N. Kaminski, L. DaSilva, I. Tinirello, G. Bianchi, F. Gringoli, A. Zubow, and M. Chwalisz. "Wireless software and hardware platforms for flexible and unified radio and network control." European Conference on Networks and Communications (Eu-CNC). 2015.

[12] I. Tinnirello, G. Bianchi, P. Gallo, D. Garlisi, F. Giuliano, and F. Gringoli. "Wireless MAC processors: Programming MAC protocols on commodity hardware." INFOCOM, 2012 Proceedings IEEE. IEEE, 2012.

[13] B. Jooris, J. Bauwens, P. Ruckebusch, P. De Valck, C. Van Praet, I. Moerman, and E. De Poorter. "Taisc: a cross-platform MAC protocol compiler and execution engine." Computer Networks (2016).

[14] S. Bouckaert, W. Vandenberghe, B. Jooris, I. Moerman, and P. Demeester. "The w-iLab. t testbed." International Conference on Testbeds and Research Infrastructures. Springer Berlin Heidelberg, 2010.

[15] J. Bauwens, B. Jooris, P. Ruckebusch, D. Garlisi, J. Szurley, M. Moonen, S. Giannoulis, I. Moerman, E. De Poorter. "Demo Abstract : Cross-technology TDMA synchronization using energy pattern beacons", CNERT, Infocom, 2017 